\title{
Glabella: A susceptible area to tissue necrosis upon injection of hyaluronic acid filler
}

\section{Kwon Osung, Chung Hyun, Park Joonsoo}

\author{
Department of Dermatology, School of Medicine, Catholic University of Daegu, Namgu, Daegu, South Korea
}

Corresponding author: Joonsoo Park, M.D., Ph.D., E-mail: g9563009@cu.ac.kr

Sir,

Filler injection has become widely used to remove the appearance of wrinkles and reduce the skin folds. Recently hyauluronic acid has become the material of the choice for filler injection. Hyaluronic acid filler has advantages in its long lasting features, low immunogenicity, and reversibility. However, despite its advantages, hyaluronic acid can cause tissue necrosis with interruption of vascular supply from injected hyaluronic acid gel [1].

A 49-year-old woman presented with erythematous patches, vesicles, ecchymosis with ulceration on the glabella and forehead for few days (Fig. 1). The lesion developed after hyaluronic acid filler injection in local hospital. The patient was not involved with any dermatologic, immunologic past history. The punch biopsy was done from the ulcerative lesion. The biopsy of the lesion showed necrotic, ulcerated epidermis with lymphohistiocytic and neutrophilic infiltration in the dermis. Extracellular basophilic amorphous materials, which highly suggest injected hyaluronic acid gels were also observed (Fig. 2). The skin lesion was improved with residual scar after treating with steroids and antibiotics for 1 month.

A considerable number of case reports are published and concluded the glabella as a 'danger area' of tissue necrosis. Paucity of collateral circulation of vascular supply in glabella makes it vulnerable to tissue necrosis. The nature of heavy bleeding druing filler injection and acute onset of skin lesion were conclusive clues to a skin necrosis caused by hyaluronic acid as presented in this case [2].

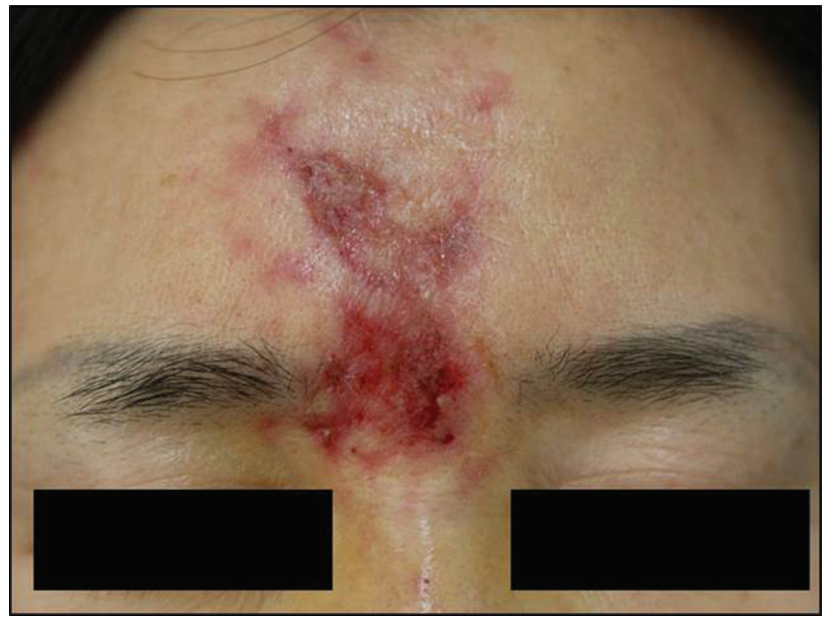

Figure 1: Clincal image of the patient presenting erythematous ulcerative patches on glabella.

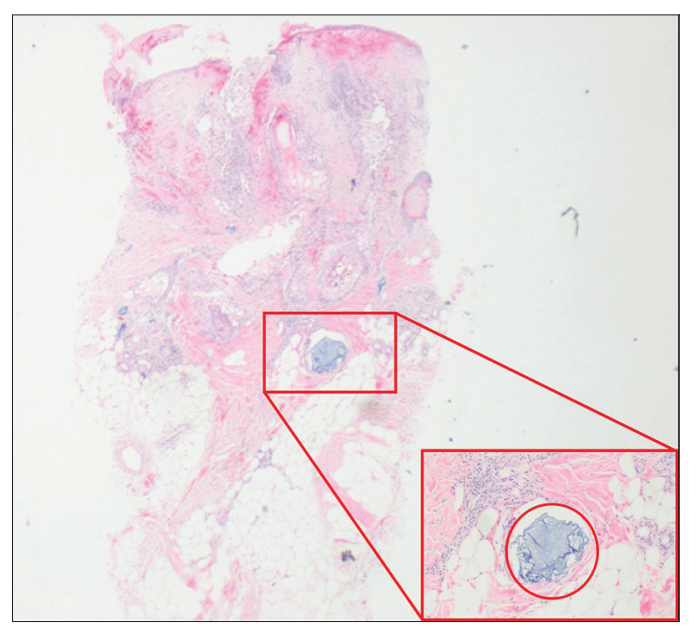

Figure 2: The histopathological examination shows ulcerative epidermis, extravasation of erythrocyte with lymphohistiocytic infiltration in dermis. Extracellular basophilic amorphous material in dermis is shown in inlet (red circle). (H\&E x20, inlet H\&E x100).

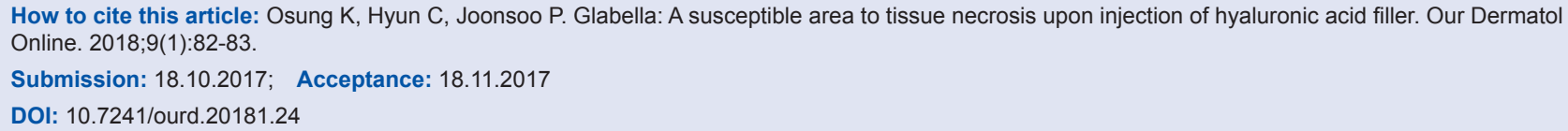


In order to prevent vascular occlusion, the depth of filler injection around glabella should remain superficial and the needle point should be directed centrally. In addition, the injection must be delivered over two to three times rather than injecting it continuously. Regurgitation of the syringe is also critical to prevent vascular injury [3]. Upon suspicion of vascular occlusion, hot compression and $2 \%$ nitroglycerin should be delivered to restore blood flow. Moreover injection of hyaluronidase and low molecular weight heparin may be conducive to relive the occluded area $[3,4]$. Therapeutic options to consider in tissue necrosis include a number of measures from hot and cold compressions, antibiotics, steroids to laser and surgical revision in severe cases. The presented case showed improvement after applying topical steroids along with oral steroids and antibiotics. As filler procedures performed by non-dermatology medical providers, inadvertent use of hyaluronic acid increases which ultimately lead to vast number of tissue necrosis. The anatomical aspects of filler injection in areas such as the glabella and tip of the nose should require more precaution and regular follow-ups as these are more susceptible to tissue necrosis.

\section{REFERENCES}

1. Park TH, Seo SW, Kim JK, Chang CH. Clinical experience with Hyaluronic acid-filler complications. J Plast Reconstr Aesthet Surg. 2011;64:892-6.

2. Grunebaum LD, Bogdan Allemann I, Dayan S, Mandy S, Baumann $\mathrm{L}$. The risk of alar necrosis associated with dermal filler injection. Dermatol Surg. 2009;35 Suppl 2:1635-40.

3. Joel L. Cohen Understanding, avoiding, and managing dermal filler complications. Dermatol Surg. 2008;34:S92-9.

4. Kassir R, Kolluru A, Kassir M. Extensive necrosis after injection of hyaluronic acid filler: case report and review of the literature. J Cosmet Dermatol. 2011;10:224-31.

Copyright by Kwon Osung, et al. This is an open access article distributed under the terms of the Creative Commons Attribution License, which permits unrestricted use, distribution, and reproduction in any medium, provided the original author and source are credited.

Source of Support: Nil, Conflict of Interest: None declared. 